\title{
Limbal and corneal calcification in patients with chronic renal failure
}

\author{
N Klaassen-Broekema, O P van Bijsterveld
}

\begin{abstract}
In patients with chronic renal failure on regular dialysis treatment, limboconjunctival degenerations and calcifications are commonly observed. In this study three groups of patients were followed over a period of 6 years. The first group consisted of 47 patients with renal failure, the second group of 17 patients with renal failure and hyperparathyroidism not controlled by drugs, and the third group seven patients with primary hyperparathyroidism without renal failure. The aim of this study was to determine the progression of the limboconjunctival changes over time. The hypothesis that an increase in serum calcium and phosphorus concentrations, as a result of tertiary hyperparathyroidism, could possibly add a corneal component to the limbal calcification was also tested. All patients with renal failure (in as much as the degenerative limbal features were not obscured by deposits of lime salts), had a type II white limbus girdle of Vogt. This limbal degeneration was observed in only $45 \%$ of controls. In all 47 patients with renal failure conjunctival calcification was observed; 26 of them also had limbal calcification. After 6 years 41 patients had developed limbal calcification. This progression was statistically significant. In 15 out of 17 patients with tertiary hyperparathyroidism a band-shaped keratopathy developed in addition to the limboconjunctival calcification.
\end{abstract}

(Brf Ophthalmol 1993; 77: 569-571)

In patients with renal failure on regular dialysis treatment, chalky white lesions can be observed clinically at the limbus. ${ }^{1}$ These have been subsequently identified as hydroxyapatite. ${ }^{2}$ The precipitation of hydroxyapatite is assumed to occur when the product of the serum concentrations of calcium and phosphorus exceeds the in vivo solubility product - that is, in a markedly supersaturated concentration. ${ }^{3}$

In renal failure, with its complexity of chemical and metabolic disturbances and because of the changes brought about by therapy, one can expect ever changing serum calcium and phosphorus concentrations with a tendency, however, to low serum calcium concentrations early in renal failure and increasing serum calcium concentrations as the disease progresses and tertiary hyperparathyroidism sets in. Tertiary hyperparathyroidism is a secondary hyperparathyroidism uncontrolled by drugs that is characterised by high levels of serum calcium, phosphorus, and parathyroid hormone.

Certain constitutional diseases such as primary hyperparathyroidism, ${ }^{4}$ and toxic states such as vitamin $\mathrm{D}$ poisoning, are also characterised by high serum calcium levels over a prolonged period of time. In these cases a band-shaped corneal degeneration and calcification develops at the level of Bowman's membrane in the exposed part of the cornea.

The aim of this study was to determine the progression of the limbal degenerations and calcifications in patients with chronic renal failure over time. We also tested the hypothesis that an increase of the serum calcium and phosphorus concentrations, as a result of tertiary hyperparathyroidism, could possibly add a corneal component to the limbal calcification.

\section{Patients and methods}

A total of 71 patients with renal failure, 47 with controllable secondary hyperparathyroidism, 17 patients with tertiary hyperparathyroidism that were scheduled to undergo surgical intervention, and seven patients with primary hyperparathyroidism but without intrinsic renal disease were evaluated for the presence and morphology of limbal and peripheral corneal degeneration and calcification.

Biomicroscopic examinations were performed using the direct and indirect illumination and scleral scatter techniques to study the difference in incidence of limbal and corneal degeneration and clinically visible calcification. Grading of the limbal calcification was done according to the system proposed by Porter and Crombie - that is, to establish the degree of calcification in patients by comparing it with drawings depicting five stages of calcification intensity. ${ }^{6}$

Grading of the corneolimbal lesions was performed at the first visit and after 3 and 6 years. The limbal calcification scores of both eyes were averaged. Serum calcium and phosphorus concentrations were determined in all patients and in 50 control subjects. The controls and the patients with primary hyperparathyroidism were not available for the second and final assessments. Linear regression, the $\chi^{2}$ enumeration statistic, and analysis of variance were used as statistical tests.

\section{Results}

\section{LIMBAL DEGENERATION}

In Table 1 the limboconjunctival and corneal changes in the patients of all groups are summarised. All patients with a Porter and Crombie ( $P$ and $C$ ) score of 2 maximally showed limbal degenerations that were seen as white incomplete arcs situated concentrically with the limbus in the interpalpebral zone. In all patients with renal failure there was, in addition, a mild to moderate calcification present. In patients with a higher $P$ 
Table 1 Limboconjunctival degenerations $(L C D)$ and calcifications (LCC) in patients with renal failure $(R F S H)$, in patients with renal failure and tertiary

hyperparathyroidism (RFTH), in patients with primary

hyperparathyroidism $(P H)$, and in healthy controls $(C O)$

\begin{tabular}{lllll}
\hline & $\begin{array}{l}\text { RFSH } \\
(n=47)\end{array}$ & $\begin{array}{l}\text { RFTH } \\
(n=17)\end{array}$ & $\begin{array}{l}P H \\
(n=7)\end{array}$ & $\begin{array}{l}\text { CO } \\
(n=150)\end{array}$ \\
\hline LCD & + & + & + & + \\
LCC & + & + & - & - \\
BSK & - & + & + & -
\end{tabular}

BSK = band-shaped keratopathy; $+=$ present; $-=$ absent .

and $\mathrm{C}$ score, the limbal degeneration was masked to a varying degree by the calcification of the limbal area but it was assumed that, also in these patients, an accompanying limbal degeneration was present. In a healthy control group of 150 patients only $45 \%$ showed these degenerations.

The degenerative opacities were located immediately beneath the epithelial surface, usually without a clear interval with the corneoscleral border. On the central edge radiating curved, off-white lines were present with a chalky aspect that appeared to be related to the terminal parts of the pallisades and limbal capillaries. In these deposits no holes were observed. These lesions are indistinguishable from the type II white limbus girdle of Vogt.

\section{LIMBAL CALCIFICATION}

In addition to the limbal degeneration, in all patients a mild or moderate calcification was present, even in the early stages of renal disease. This calcification was located in or rather over the area of degeneration. Limbal calcium salts were visible as a semilunar arc of delicate crystalline deposits adjacent to the scleral border. As the disease progressed, the degree of limbal calcification increased with the formation of coarse to very coarse amorphous plaques in some cases. Table 2 gives the degree of limbal calcification at the initial assessment, after 3 , and after 6 years for patients.

In two cases of marked limbal calcification, the amorphous plaques extended on to the corneal tissue just beyond the central limbal rim. The calcific precipitates, fading in the central direction of the cornea, were shown biomicroscopically to be situated between the basal layers of the epithelium and the membrane of Bowman. These concretions occasionally flake, leaving painful corneal erosions that stained with fluorescein and a localised area of hyperaemia.

Table 2 Limbal calcification in 47 patients with renal failure and on regular haemodialysis but without tertiary hyperparathyroidism, at the initial evaluation, and after 3 and 6 years, graded according to the system proposed by Porter and Crombie. The scores were averaged over the right and left eye and over the nasal and temporal corneal section

\begin{tabular}{lrrrrr}
\hline & \multicolumn{7}{l}{ Grade of calcification } \\
\cline { 2 - 6 } Assessment & 1 & 2 & 3 & 4 & 5 \\
\hline Initial & 21 & 15 & 8 & 3 & 0 \\
After 3 years & 10 & 18 & 12 & 5 & 2 \\
After 6 years & 5 & 14 & 15 & 8 & 4
\end{tabular}

*One patient died before the final assessment.

$\chi^{2}[8]=20, p=0 \cdot 01$

\section{DEGREE OF CALCIFICATION}

There is a clinical impression that the intensity and the coarseness of the calcification was related to the calcium and phosphorus product. However, no association could be demonstrated between the degree of calcification expressed in the $P$ and $C$ grading system and the serum calcium levels $(r=+0.02)$, the serum phosphorus levels $(r=+0.17)$, or with the serum calcium and phosphorus product $(r=+0 \cdot 20)$ in 47 patients with renal failure without tertiary hyperparathyroidism.

\section{PROGRESSION OF THE LIMBAL CALCIFICATION}

The progression of the limbal calcification was on average slow but statistically significant $(p=0 \cdot 01)$. Three years after the initial assessment, the limbal calcification had increased by slightly more than one half grading point on average. However, in some patients a rather rapid progression was noted that could be as much as three grading points in the final assessment; conversely in some other patients the progression was almost imperceptible.

The association between the degree of calcification and the duration of dialysis (years) is statistically significant $(p<0.05)$, but this association is weak: the product moment correlation coefficient is $+0: 30$.

\section{BAND-SHAPED KERATOPATHY}

In 15 of 17 patients with renal failure and tertiary hyperparathyroidism, in addition to calcific deposits in the limbus, calcification of a different type and localisation was observed. In two of these 15 patients the opacity was unilateral. This type of calcification presented itself as opacities with a soft grey colour showing biomicroscopically a subtle granular structure. They were visible approximately $1 \mathrm{~mm}$ from the corneoscleral junction on both the nasal and temporal side of the cornea and extended axially with an ill defined central border. In the greyish area dark round holes were distinguishable (Fig 1).

These axial corneal degenerations were indistinguishable from band-shaped keratopathy. Also all seven patients with primary hyperparathyroidism showed identical corneal calcifications. Band-shaped keratopathy was

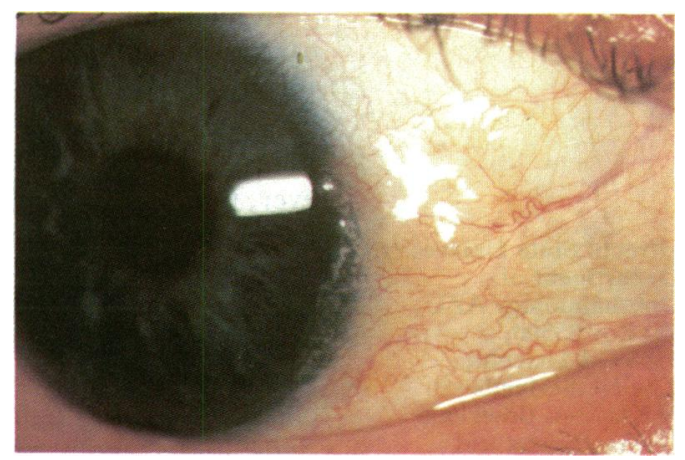

Figure 1 Early band-shaped keratopathy. In this stage the calcium and phosphorus product is markedly elevated because of tertiary hyperparathyroidism. Note also the coarse white limbal calcification. 
Table 3 The average values and the standard deviations of serum calcium and the serum phosphorus concentration as well as their product in patients with renal failure and secondary hyperparathyroidism (RFSH), with renal failure complicated by tertiary hyperparathyroidism $(R F T H)$, with primary hyperparathyroidism $(P H)$, and in controls $(C O)$

\begin{tabular}{|c|c|c|c|c|c|c|c|}
\hline & \multirow[b]{2}{*}{ No } & \multicolumn{2}{|c|}{ Serum calcium } & \multicolumn{2}{|c|}{ Serum phosphorous } & \multirow{2}{*}{$\begin{array}{l}\text { Average } \\
C a^{\star} P\end{array}$} & \multirow{2}{*}{$\begin{array}{l}S D \\
C a^{\star} P\end{array}$} \\
\hline & & Average & $S D$ & Average & $S D$ & & \\
\hline $\begin{array}{l}\text { RFSH } \\
\text { RFTH } \\
\text { PH } \\
\text { CO }\end{array}$ & $\begin{array}{r}47 \\
17 \\
7 \\
50\end{array}$ & $\begin{array}{l}2.46 \\
2 \cdot 70 \\
3.33 \\
2 \cdot 41\end{array}$ & $\begin{array}{l}0.23 \\
0.28 \\
0.48 \\
0 \cdot 10\end{array}$ & $\begin{array}{l}1 \cdot 87 \\
2 \cdot 17 \\
0.96 \\
1.30\end{array}$ & $\begin{array}{l}0.64 \\
0.68 \\
0.17 \\
0.17\end{array}$ & $\begin{array}{l}4.52 \\
5.82 \\
3 \cdot 19 \\
3 \cdot 13\end{array}$ & $\begin{array}{l}1.33 \\
1.78 \\
0.77 \\
0.46\end{array}$ \\
\hline
\end{tabular}

observed in only two of the 47 patients with secondary hyperparathyroidism and renal failure. One of these two patients suffered from long standing renal insufficiency complicated by amyloidosis and the other patient had a serious diabetic nephropathy.

The serum calcium concentrations differed significantly between the patients of the various groups but not between those with renal failure complicated by secondary hyperparathyroidism and the controls. The serum phosphorus concentrations also differed significantly between patients and controls but not between patients with secondary and tertiary hyperparathyroidism (Table 3).

\section{Discussion}

There have been different interpretations of the similarity and the relationship between Vogt's white limbus girdle and the limbal calcification in renal failure. ${ }^{18-10}$ In patients in which the effects of renal failure in one eye or in one part of the eye preceded the contralateral side, our observed sequence of events was the development of degeneration, indistinguishable from the white limbus girdle type II, followed by calcification, initially as the deposition of a semicircular zone of delicate crystals and later as coarse amorphous calcific plaques as the metabolic situation deteriorated. The deposition of lime salts in the conjunctiva and the limbus may be related to the ability of these tissues to react with elastosis to uraemia."

Calcium salts can be deposited in a suitable matrix if the concentration of calcium and phosphorus exceeds the in vivo solubility product. This form of calcification in undamaged tissues is referred to as metastatic calcification. There is an increasing body of experimental evidence, however, summarised and amplified by Scarpelli, ${ }^{12}$ that demonstrates that tissue damage always precedes calcification. Also clinically ${ }^{13}$ it has to be remembered, that in healthy persons there is no calcium deposition in spite of the fact that the interstitial fluids are supersaturated with calcium and phosphorus. ${ }^{14}$ In healthy persons the precipitation of calcium salts is usually prevented by protective serum proteins.

The production of a calcifiable matrix is necessary for the deposition of calcium salts. ${ }^{15}$ Elastosis which precedes calcification could induce the formation of a calcifiable matrix but it is not in itself a matrix, as calcification has never been reported within the elastotic tissue itself. ${ }^{1013}$ Calcification of the outer eye has a predilection for the immediate subepithelial site as it is believed that loss of carbon dioxide to the atmosphere occurs rapidly on the surface of the eye in the interpalpebral area. ${ }^{8}$ The fall in $\mathrm{pCO}_{2}$ results in a rise of $\mathrm{pH}$. Hydroxyapatite is less soluble in an alkaline environment so that these lime salts are precipitated in areas with a relatively high alkalinity.

In renal failure there is a rise in the serum phosphorus concentration because of decreased reabsorption causing the serum calcium concentration to fall reciprocally. The net result, however, is an increase of the product of the serum calcium and phosphorus concentration. Under these conditions there is a chalky white precipitation of lime salts in the conjunctiva and limbus. As the disease progresses there is increased activity of the parathyroid glands that can result in an increase in serum calcium levels leading to a different type of calcification. This corneal calcification is very similar to the band-shaped keratopathy of primary hyperparathyroidism. In primary hyperparathyroidism band-shaped keratopathy seems to be associated, among other things, with high serum calcium concentrations. In patients with renal failure complicated by tertiary hyperparathyroidism the serum calcium concentrations are significantly higher than in patients with renal failure and secondary hyperparathyroidism and this may be responsible for the development of band-shaped keratopathy in patients with tertiary hyperparathyroidism.

We are grateful to Dr J Vos, nephrologist in the Foundation for Home Dialysis in Utrecht, for permission to examine patients under his care and for his valuable information.

1 Abrams JD. Corneal and other findings in patients on intermittent dialysis for renal failure. Proc Roy Soc Med 1966; 59. tent dialys

2 Berkow JW, Fine BS, Zimmerman LE. Unusual ocular calcification in hyperparathyroidism. Am $\mathcal{F}$ Ophthalmo $1968 ; 66: 812-4$

3 Stanbury SW. Bony complications of renal disease. In: Black DAK, ed. Renal disease. Oxford: Blackwell, 1962: 508-52.

4 Cogan DG, Albright F, Bartter FC. Hypercalcemia and band keratopathy. Arch Ophthalmol 1948; 40: 624-38.

5 Meesman A. Hypokalzaemie und Linse: ein Beitrag zur Behandlung der Tetanie und der Cataracta tetanica mit AT 10 Holtz. Klin Monatsbl Augenheilkd 1938; 100: 1-66.

6 Porter R, Crombie AL. Corneal and conjunctival calcification in chronic renal failure. Br $\mathcal{F}$ Ophthalmol 1973; 57 : 339-43.

7 Klassen-Broekema N, Bijsterveld OP van. Red eyes in renal failure. Br f Ophthalmol 1992; 76: 268-71.

8 Berlyne JW, Shaw AB. Red eyes in renal failure. Lancet 1967 i: $4-7$

9 Berlyne JW. Microcrystalline conjunctival calcification in renal failure. Lancet 1968; ii: 366-70.

10 Ehlers N, Kruse Hansen F, Hansen HE, Jensen OA. Corneoconjunctival changes in uremia. Acta Ophthalmol 1972; 50 83-94.

11 Finlayson GR, Smith G, Moore MJ. Effects of chronic acidosis on connective tissue. $\mathcal{F A M A} 1964 ; 187$ : 659-62.

12 Scarpelli DG. Experimental nephrocalcinosis, a biochemical and morphologic study. Lab Invest 1956; 14: 123-41

13 Klaassen-Broekema N, Landesz M, Krul B, Bijsterveld OP van. Metastatic or dystrophic conjunctival calcification in van. Metastatic or dystrophic conjunctival calcific
renal failure? Eur 7 Ophthalmol 1992; 2: 150-4.

14 Neuman WF, Neuman MW. In: The chemical dynamics of bone mineral. Chicago: University of Chicago Press, 1958.

15 Eisenstein R, Truchart RE, Hass GM. Pathogenesis of abnormal tissue calcification. In: Sognaes RF, ed. Calcification in biological systems. Washington DC: American Association for the Advancement of Science 1960: 281-305. 